\title{
Políticas de gestión de la educación pública escolar en Chile (1990 -2010): una evaluación inicial
}

— Sebastián Donoso*

Gonzalo Donoso**

\section{Resumen}

El artículo analiza las políticas de gestión de la enseñanza obligatoria en Chile al cumplirse próximamente dos décadas del proyecto educacional vigente, el cual ha logrado progresos importantes en materia de cobertura e incremento presupuestario, pero no han sido equivalentes sus avances en la calidad de la educación, pese a que en las comparaciones internacionales Chile mejora su posición relativa. El texto levanta un diagnostico del problema, cuyo núcleo central está constituido por la tensión entre la lógica del mercado con la cual opera el financiamiento escolar y la lógica de los derechos ciudadanos de educación que se ha buscado afanosamente implantar. Este camino está prácticamente agotado y tras dos décadas de forzar su andar demanda un cambio profundo en la manera cómo se diseña e implementan las políticas de gestión de educación obligatoria en Chile. El artículo releva el peso de la desigualdad social en el logro de los objetivos pedagógicos del sistema escolar público chileno, analiza las dificultades institucionales y, finalmente, evalúa algunos puntos críticos, generando proposiciones para la búsqueda de soluciones. Palabras clave: Gestión de la enseñanza obligatoria. Evaluación de la gestión escolar. Políticas de le gestión de la enseñanza pública en Chile. Desafíos de la gestión educacional.

\section{Políticas de gestão da educação pública escolar no Chile (1990-2010): uma avaliação inicial Resumo}

0 artigo analisa as políticas de gestão do ensino obrigatório no Chile ao se cumprir aproximamente duas décadas do projeto educacional vigente que logrou progressos importantes em matéria de cobertura e implementação, porém

\footnotetext{
* Sebastián Donoso, Doctor en Educación; Académico del Instituto de Investigación y Desarrollo Educacional, Universidad de Talca, e Investigador Asociado del Centro Avanzado de Investigaciones en Educación (CEIA-CONICYT). E-mail: sdonoso@utalca.cl

** Gonzalo Donoso, Sociólogo (Pontificia Universidad Católica de Chile); Magistrante en Gerencia y Gestión Pública (Universidad de Chile).E-mail: gfdonost@puc.cl
}

Ensaio: aval. pol. públ. Educ., Rio de Janeiro, v. 17, n. 64, p. 421-448, jul./set. 2009 
não foram equivalentes seus avanços em relação à qualidade da educação mesmo sabendo-se que nas comparações internacionais o Chile melhora sua posição relativa. 0 texto faz um diagnóstico do problema, cujo núcleo central está constituído pela tensão entre a lógica do mercado com a qual opera o financiamento escolar e a lógica dos direitos à educação que se buscou implantar. Este caminho está praticamente esgotado e depois de duas décadas de tentativas demanda uma mudança profunda no modo de planejar e implementar as políticas de gestão da educação obrigatória no Chile. 0 artigo releva o peso da desigualdade social para o alcance dos objetivos pedagógicos do sistema escolar público chileno, analisa as dificuldades institucionais e, finalmente, avalia alguns pontos críticos, gerando proposições para a busca de soluções.

Palavras-chave: Gestão do ensino obrigatório. Avaliação da gestão escolar. Políticas de gestão do ensino público no Chile. Desafios da gestão educacional

\section{Management policy of the public school education in Chile (1990 -2010): an initial assessment Abstract}

This article discusses the compulsory education management policies in Chile assembled in the next two decades of the current educational project, which has achieved significant progress in terms of coverage and budget increase, but it has not had the same progress in the quality of education, although Chile had improved its international position. The text raises a diagnostic problem, which core consists of the tension between the logic of the market that finances the school and the logic of education as a civil right which they eagerly sought to implement. This road is almost dried after two decades of demanding changes in the way they design and implement the compulsory education management policies in Chile. This article highlights the weight of social inequality in achieving the educational objectives of the Chilean public schools system, analyzes the institutional difficulties, and finally assesses some critical points to generate proposals to create solutions.

Keywords: Compulsory education management. Evaluation of school management. Management policy is the public education in Chile. Challenges for the education management.

\section{Introducción}

El artículo analiza las políticas de gestión de la enseñanza obligatoria en Chile a partir del retorno de la democracia (1990), cumplidas prácticamente dos décadas de continuidad democrática y también de la coalición gobernante:

Ensaio: aval. pol. públ. Educ., Rio de Janeiro, v. 17, n. 64, p. 421-448, jul./set. 2009 
Concertación de Partidos por la Democracia, situación que marca un punto distintivo importante en materia de continuidad en la historia de la política educativa chilena del siglo XX, incluso con el pasado reciente de los casi 18 años de dictadura cívico/militar en Chile, que pese a la continuidad formal, estuvo marcada por orientaciones que en momentos fueron opuestas.

Al cumplirse próximamente dos décadas del proyecto educacional vigente, es momento para establecer una evaluación inicial de este proceso, el cual, además se vio reforzado por la puesta en marcha de una reforma educativa que tuvo impactos relevantes -al menos en el destino de recursos financieros para el sector-algo que desde hace mucho tiempo no se registraba en el volumen y frecuencia establecido en estos años'.

La comprensión de este tema en el caso chileno requiere de precisiones iniciales que contribuyen a identificar las transformaciones del sistema educacional en los últimos 40 años, y permiten identificar su impacto en la gestión e implementación de la educación pública. En este lapso de tiempo, Chile experimentó cambios significativos en sus políticas educacionales que se han traducido también a los procesos de gestión. Desde inicios de los años 80 los establecimientos de enseñanza pública son gestionados directamente por el Estado local, estableciéndose un régimen descentralizado en esta materia que opera con el financiamiento vía subsidio a la demanda. De esta forma, los centros públicos dependen de los gobiernos locales (denominándose enseñanza municipal) como también existen centros que son gestionados por empresarios privados que cumplen con criterios de educación pública (denominados Particular Subvencionado).

La tarea de universalizar la educación primaria no ha sido fácil para Chile, y solo después de 60 años de aplicación de políticas al respecto se puede señalar que desde los años 80 se cumplió debidamente con ella (8 años). En segunda instancia, a partir del año 2003 se define la obligatoriedad de la enseñanza secundaria (4 años), proceso que ha tenido una fuerte expansión pero que aún no registra plena cobertura (92\%), especialmente por los problemas endémicos de deserción que afectan a la población escolar más vulnerable de ese nivel de enseñanza.

La visión asumida en el trabajo dice relación, en primer lugar, con el hecho que la política de gestión de la educación no es constitutiva sino tributaria de la política educacional, por ende, analizarle como fenómeno desvinculado implica una visión sesgada que deriva en sostener que los principios y mecanismos de gestión son

\footnotetext{
'Durante los años 80, bajo el gobierno de Pinochet, los recursos públicos para el sector se redujeron en términos reales en un 24\% (COX, 2003). Es decir lejos de invertir en este sector, la dictadura se caracterizó por cortar recursos y en forma paralela implementar otras medidas que incidieron e inciden aún significativamente en la carga negativa que tiene la gestión educacional en el país.
}

Ensaio: aval. pol. públ. Educ., Rio de Janeiro, v. 17, n. 64, p. 421-448, jul./set. 2009 
neutrales, algo que se contradice en si mismo, ya que toda política busca dar orientaciones para direccionar los resultados que se pretende alcanzar. Además, la política de gestión también implica referirse a dos aspectos centrales que conforman su base de sustentación: el financiamiento de la educación obligatoria y el soporte legal, ambos son parte relevante de la política general de educación.

El texto expone, en primer lugar, el análisis de los principales cambios experimentados en las políticas de gestión de la educación en los años en estudio, identificando los problemas relevantes del sector y su impacto en la educación pública. A partir de ello, se realiza un análisis de los sistemas de soporte a los procesos de gestión y su evolución en el tiempo, atendiendo esencialmente aquellos ocurridos durante la presente década (2000) orientados a corregir las dificultades más severas que ha enfrentado el sistema público. En tercer lugar, se revisa el marco de adecuaciones implementado por los establecimientos escolares para cumplir sus objetivos $y$, finalmente, se lleva acabo un debate evaluativo de lo ocurrido y de algunas disyuntivas que enfrenta la política de gestión de la educación pública chilena.

\section{El diagnóstico del problema: la gestión de los establecimientos escolares}

En Chile, la gratuidad de la enseñanza primaria obligatoria es uno de los pocos principios que desde inicios del siglo XX que se ha mantenido vigente tras su proclamación en el año 1860, siendo un tema sustentado -básicamente- en la contribución de la educación a la promoción del crecimiento y la equidad en la sociedad, y en su asimilación a un bien de carácter público. Consistente con ello, el gasto público es y ha sido la principal fuente de financiamiento de la educación obligatoria, y si bien participa el sector privado en esta función, lo hacen con recursos públicos².

Desde el punto de vista de la política pública, la fractura de la democracia chilena ocurrida el año 1973 implicó el rediseño de la sociedad en sus diversos ámbitos. Siendo el sector educación el que se ajustó más tardiamente al modelo de economía de mercado neoconservador impulsado en Chile desde el año 1976, debido a que en el seno de la misma Dictadura cívico/militar existían indecisiones y disputas entre dos corrientes una ligada a la visión más tradicional del Estado, con un fuerte rol del sector público en la provisión de educación, y otra-que finalmente es la dominante-que propugnaba decididamente su apertura a la privatización, lo que derivó en cambios de gran relevancia en las políticas de gestión y de los instrumentos de financiamiento de la educación pública en todos sus niveles.

2 Al año 2006 el sistema escolar chileno, desde el nivel preescolar al secundario, está conformado por algo más de 3,6 millones de estudiantes, cifra que se encuentra estabilizada desde el año 2002 con variaciones menores (CHILE, 2007). 
La reforma al sistema de financiamiento de la educación pública aplicada a partir del año 1981 fue una de las transformaciones más eficaces impulsadas en el sector educación en los últimos 40 años, por cuanto no solamente incidió en los criterios, orientaciones y mecanismos de financiamiento, sino que también posee aún impactos relevantes en la gestión pedagógica del centro escolar, de forma que se trató de una reforma educacional impulsada desde las política de financiamiento del sector. La nueva política de financiamiento público asume la demanda por educación como el criterio central en la asignación de los recursos. Se aplicó masivamente a partir del año 198133, significando cambios en la arquitectura del sistema en tres aspectos claves, a saber: primero, un proceso generalizado de descentralización de los establecimientos escolares -que en su totalidad pertenecían al Estado nacionallos cuales fueron transferidos a los gobiernos locales (Municipios). Segundo, de igual forma los docentes y personal no docente fueron transferidos a los gobiernos locales, perdiendo un conjunto relevante de beneficios económicos y de carrera profesional que incluso a la fecha aún no recuperan del todo, pese a los reconocidos esfuerzos desplegados por los gobiernos democráticos. El tercer componente clave es que se estimuló y facilitó la libre entrada/salida de proveedores privados de educación, mediante los nuevos instrumentos de gestión financiera en operación.

Un informe OCDE (OCDE; CHILE, 2004, p. 186) sobre el sector educación en Chile concluye que "basarse en mecanismos de mercado (competencia entre escuelas y pago de incentivos a los docentes) para producir en el futuro una mayor efectividad y eficiencia en el sistema educacional no es una estrategia de alto rendimiento". De alli la relevancia que adquieren en el escenario chileno los "programas compensatorios", como estrategia de gestión que se viene implementando desde 1990 a la fecha, busca transformarse en una nueva agenda de gestión pública centrada en los objetivos de calidad y equidad en educación, pese a que sus éxitos han sido relativos. Mediante el impulso de diversas iniciativas se persigue que el Estado asuma un rol más amplio, responsabilizándose de aspectos que el modelo subsidiario no considera. Sin embargo, esta tarea se enfrenta a una limitante estructural no resuelta a la fecha. El Ministerio de Educación aunque posee la tuición técnica sobre los establecimientos educacionales, no puede - sino que menguadamente intervenir, ni tampoco direccionar la acción pedagógica de ellos o de los empresarios, los directivos y sus establecimientos educacionales.

Es decir, el sistema posee un vicio operacional que no permite al Ministerio la implementación de medidas que garanticen la provisión de una educación de

\footnotetext{
${ }_{3}^{3}$ Si bien el proceso se completa el año 1987, más de la mitad de los establecimientos escolares son traspasa dos ese año a este nuevo sistema de financiamiento, y de paso todo el sistema universitario público también a una nueva modalidad.
}

Ensaio: aval. pol. públ. Educ., Rio de Janeiro, v. 17, n. 64, p. 421-448, jul./set. 2009 
calidad, al que denominados 'enfoque matricial' para efectos de identificarle debidamente. Este problema es el tema central de gestión de la educación pública, resuelto parcialmente por la reciente Ley General de Educación, pero sin llegar efectivamente a una solución de fondo que permita dirimir entre los dos principales principios en conflicto en el sistema educativo chileno: "libertad de enseñanza y derecho a una educación de calidad", cuando se trata de disponer de medidas eficientes que permitan a la autoridad garantizar una educación de calidad para toda población.

Esta situación evidencia la compleja mezcla que conforma el marco de acción del Estado chileno en el diseño e implementación de las políticas educacionales. Lo que implica mezclar acciones basadas en dos paradigmas:

Una es la noción de que los mercados educacionales, con competencia entre escuelas, un alto grado de elección de los establecimientos educacionales por parte de los padres, y una administración privada de dichos establecimientos, proporcionan la mejor esperanza de de eficiencia educacional y el rendimiento escolar más alto posible. La otra es la noción de que el gobierno central debe intervenir en el sistema educacional con una clara visión de lo que constituye una buen educación y cómo lograrla para asegurar que los estudiantes tengan la mayor oportunidad de aprender (OCDE; CHILE, 2004, p. 289).

"[...] Resultando de este conflicto ideológico, reformas educativas con un débil nexo con la práctica escolar y con el sistema de formación inicial docente" (OCDE; CHILE, 2004, p. 321).

La explícita alusión a la Reforma Educativa (impulsada desde el año 1996), responde al hecho histórico derivado de la fuerte presión que el Banco Mundial y otras agencias multilaterales de desarrollo hicieron desde finales de la década de los 80, para que los países de Latinoamérica suscribieran reformas educativas de segunda generación ${ }^{4}$ (orientadas a la calidad), bajo un mismo formato operacional.

Como se ha señalado, en el caso chileno, los procesos de gestión estuvieron marcados por la complejidad de implementar medidas compensatorias, en grado importante orientadas a corregir los efectos del mercado, en una sociedad con alta desigualdad social y económica, sin cambiar las orientaciones marco de las políticas de mercado. Esta situación caracteriza con fuerza la primera etapa de los cambios del sistema educativo tras el retorno a la democracia (1990 a 1995), que además permitieron generar las condiciones para la implantación-más tarde- de

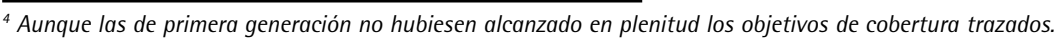


la reforma educativa (DONOSO, 2005). En este periodo se generaron las condiciones para un mejor funcionamiento del sistema escolar; con énfasis en inversión en infraestructura, materiales educativos y mejoramiento de las condiciones laborales de los docentes. Los dos criterios articuladores del conjunto de las políticas educacionales de los 90' que especifican la naturaleza del accionar público en educación son: "programas integrales de intervención de cobertura universal para el mejoramiento de la calidad de los aprendizajes y programas compensatorios focalizados en las escuelas y liceos de menores recursos con bajos resultados de aprendizaje para el mejoramiento de la equidad" (OCDE; CHILE, 2004, p. 20-21).

Sin embargo, en este ciclo (1990 -1995) es en lo financiero donde se evidencian mayores cambios: alza del valor de las subvenciones escolares; creación de la subvención especial por ruralidad, sistema de financiamiento compartido (1994).También se incrementa el porcentaje del PIB destinado a educación y sube el gasto público en educación del 12,5 al 15,3\% (OCDE; CHILE, 2004, p. 16).

Paralelamente el gasto público en educación por alumno se elevó un 23\% para la enseñanza superior, un 42\% en la enseñanza media y un 65\% en la básica (CHILE, 2002, p. 41). En los años considerados, estas cifras fueron gradualmente generando impactos positivos en los indicadores de rendimiento y comportamiento general del sistema escolar en un amplio espectro de dimensiones. Si bien la cobertura del sistema prácticamente se mantuvo estable, mejoraron los indicadores de aprobación, afectando positivamente a repitencia y abandono.

En el período siguiente (1996 -2000) que a bien se suscribe el argumento que la Reforma Educativa representa una continuación de la política educacional iniciada en los 90', la Reforma centra su eje en la transformación curricular en todos sus niveles, a la cual se suma una línea de refuerzo de la profesionalización docente, que incluye aumento sistemático de remuneraciones, incorporación de incentivos, pasantías en el exterior, perfeccionamiento fundamental, cambios en la formación inicial y premios de excelencia. Este proceso se dio en paralelo con un incremento significativo de los recursos financieros del sector. Ello también implicó incrementar el gasto público por alumno en esos años, creciendo respecto de 1995 más de un 40\% en enseñanza básica y 50\% en la media (CHILE, 2002, p. 41). En síntesis, es claro que la inversión tanto privada como pública del país en educación se elevó sostenida y significativamente, el aumento porcentual revertía el bajo gasto en educación que se alcanzó en la década del 80 e incluso superó la tasa histórica de gasto de Chile en educación (7\% del PIB) antes de la dictadura militar.

La escuela como centro de la acción corresponde a una visión estratégica que el Ministerio adopta también en lo programático, como una forma de "romper" la inadecuación del enfoque matricial 'Ministerio- Municipios', definido con 
antelación que no permite al Ministerio un adecuado control del hacer escolar. Los resultados registrados entre los años 1995 y 2000 en los indicadores clásicos del sistema educativo se desplazaron positivamente. La cobertura de la educación básica subió al 97\%, y en la enseñanza media al 84\% (CHILE, 2002, p. 25), la tasa de deserción en básica se redujo al 1.7\% y al 6.6\% en enseñanza media (CHILE, 2002, p. 33), de igual forma, la tasa de alumnos aprobados en enseñanza básica subió al 95.7\% y en la media al 89.4\% (CHILE, 2002, p. 77-78). Este proceso también implicó un mejoramiento de la cobertura de la educación preescolar, superando el 33\% de la población, aunque con una claro sesgo socioeconómico (HERRERA; BELLEI, 2002). Paralelamente la tasa bruta de participación en la educación superior sube de 28 a 34\% (BRUNNER; ELACQUA, 2003, p. 87).

Sin embargo, el impacto de los resultados reseñados sobre los aprendizajes de los alumnos en el sistema escolar no tiene la progresión esperada ante los insumos incorporados

[...] hay una tendencia consistente aunque leve, de incremento en los promedios nacionales en la primera mitad de la década, reduciendo asi levemente las diferencias de logros entre establecimientos municipales y particulares $p a-$ gados. Esta se estancó de 1996 a 2002 y la diferencia aumentó. Segundo, que la distribución social de los aprendizajes exhibe una repartición altamente estratificada e inequitativa, similar a la de 1990. Tercero, que las mejoras en rendimiento son mayores que las del promedio en el caso de las escuelas básicas que han sido objeto de programas focalizados como el P-900 o el Programa rural, lo que ha significado disminución de las brechas de rendimiento entre este alumnado -el más pobre- y el resto del país. Por último, que las diferencias de logros en el aprendizaje entre las distintas dependencias del sistema subvencionado ( $\mathrm{Mu}$ nicipal y Particular) son mínimas y no siempre favorables a la educación privada, cuando se comparan grupos socioeconómicos homogéneos (OCDE; CHILE, 2004, p. 39-40).

El principal factor explicativo de los resultados escolares son los antecedentes socioeconómicos de la familia, variable que ha sido y sigue siendo dominante en la actualidad, al extremo que relega otras -como dependencia del establecimiento- con contribuciones marginales sobre el tema. Adicionalmente, esta relación evidencia que la educación tiene un papel limitado en la reducción de la inequidad y desigualdad. Lo que se refuerza en segundo lugar, pues el estancamiento de los resultados responde también al deterioro sino al aumento de la brecha de inequidad en la distribución del ingreso en Chile, hecho que si bien ha implicado cierta reducción de la pobreza, las diferencias entre los menores y los mayores ingresos se han incrementado (CHILE, 2004). 
El estancamiento de la educación chileno en materia de logros escolares es extensivo al momento actual. Pese a que los resultados del año 2008 mostraron un avance marginal (aunque significativo) en lengua materna, se puede señalar que el "no incremento" de los puntajes en las pruebas nacionales es prolongable a las comparaciones internacionales de la Prueba PISA, más allá que ha mejorado el posicionamiento relativo del país, en las comparaciones relevantes el tema es del "no avance". Aún cuando los resultados en materia de logros no han seguido la progresión positiva que se esperaba, a la fecha continúan incrementándose los recursos financieros del sector-pese alas críticas acerca de la efectividad de estas medidas. En materia de los indicadores clásicos de eficiencia interna (retención, repitencia, deserción) el sistema educacional mantienen su tendencia positiva, pero el principal problema del sistema educacional chileno - como se ha visto- es que no logra traducir la eficiencia interna en avances relevantes de eficiencia externa.

Los resultados expuestos en esta sección muestran claramente que la calidad de la educación es la temática pedagógicamente dominante de una reforma que habiendo cumplido un ciclo importante en metas de cobertura enseñanza primaria y secundaria, y por ende de integración al sistema escolar de la población más vulnerable, no ha podido resolver con igual eficiencia la provisión de una educación de calidad. También queda en evidencia que la implementación de una educación de calidad-se asienta en el caso chilenosobre una base social extraordinariamente compleja de desigualdad que se manifiesta en el ámbito social, económico, cultural y finalmente en el educativo

Los argumentos destacados se traducen en un conjunto de problemas claves que inciden en forma gravitante sobre la gestión de los establecimientos, a saber:

- Un sistema de financiamiento incompatible con las tareas de largo plazo que asume la educación. Ello ocasiona no solamente severas dificultades de financiamiento de los sistemas escolares que trabajan con la población más vulnerable, sino que también una gran incertidumbre en las tareas de mediano y largo plazo.

- Una alta heterogeneidad social, reforzada además por la gran segregación territorial como también, por la resultante de un sistema de financiamiento que no ha sido capaz de establecer valores de subsidio asociados a los costos reales de la formación de la población más vulnerable, derivando en un incremento de la homogeneidad social al interior de cada establecimiento pero en un aumento de la heterogeneidad respecto de otros, provocando severas dificultades de socialización (construcción de un pacto social incluyente) ${ }^{5}$.

\footnotetext{
5 La resultante es una reducción de la varianza de capital social intra establecimientos y un aumento de la inter establecimientos, dando cuenta de mayor diferencia social.
}

Ensaio: aval. pol. públ. Educ., Rio de Janeiro, v. 17, n. 64, p. 421-448, jul./set. 2009 
- Dificultades relevantes para implementar alianzas y estrategias de largo plazo entre diversos actores intra-inter sistema educativo, producto del proceso de concesiones y licitaciones implementado bajo mecanismos de mercado. Siendo concordante con la visión de Estado subsidiario, el Ministerio no puede generar alianzas estratégicas con socios estables en los distintos procesos que debe afrontar (BECA, 2006, p. 30).

- Escasa autonomía de los centros escolares municipales y de sus cuadros directivos para asumir decisiones relevantes en materia de selección, contrato y cambio de docentes, de manejo de recursos financieros, etc.

- Aumento de visiones tecnocráticas de la gestión, desligadas de la práctica educativa de los centros escolares. Se enfatiza la eficiencia en el manejo de los recursos como clave del éxito como un factor independiente del capital social de los estudiantes y sus familias y de las condiciones del trabajo laboral docente, atribuyéndose a los privados la mayor eficiencia, más que a las condiciones bajo las cuales éstos trabajan.

Al conjunto de problemas expuestos debe sumarse la falta de una vinculación efectiva entre los procesos de formación inicial de docentes, la carencia absoluta de una carrera docente que articule las demandas laborales, las de formación continua y los mecanismos de retribución económica y social. En la actualidad no hay mecanismos eficientes, legales ni financieros, para articular e integrar al poderoso sector empresarial particular que provee educación pública, en una acción común y compartida con los agentes estatales, que permita el funcionamiento de una carrera docente que vincule la formación, perfeccionamiento y progresión en la profesión. Como señala Pavez (2003), es difícil justificar que los regímenes laborales y de remuneraciones de los maestros que se financian con el mismo tipo de subsidio público sean tan desiguales según corresponda el empleador a un agente privado o público.

Las implicancias de lo señalado sobre el mercado laboral docente se reconocen en diversos trabajos (MIZALA; ROMAGUERA, 2003; RAMA; NAVARRO, 2004; ROJAS, 1998), compartiendo el argumento que el mercado educativo es un conjunto de submercados o mercados diferenciados, más que un solo mercado. Se trata de realidades acotadas que tienen a funcionar entrópicamente con ciertas reglas de mercado, pero con adecuaciones importantes. Las barreras de ingreso de nuevos docentes al sistema son bajas. La exigencia de acreditación de eficiencia profesional (calidad) por el profesor se remite a la certificación del título profesional y, fundamentalmente, responde a la decisión de disponibilidad de recursos financieros del establecimiento (plaza) para contratarle.

Adicionalmente, la movilidad laboral transversal en el mercado (cambio de institución) también es baja. Los maestros privilegian estabilidad laboral versus 
el aumento de sus ingresos económicos (MIZALA; ROMAGUERA, 2003, p. 549). Por su parte la movilidad horizontal es aún menor, no existe un sistema organizado de carrera funcionaria, regional o nacional, lo que reduce la posibilidad de articular criterios comunes en este plano. Incluso en referencia a los cargos directivos, la nueva ley de renovación de directivos del ámbito municipal que debió aplicarse masivamente a partir del año 2006, no tuvo el soporte financiero requerido para operar conforme fue definida, teniendo ala fecha una palicación auna escala menor a la proyectada.

El silencio en materia de una política pública de formación continua de maestros ha sido suplido deficientemente en la actual década por políticas parciales con limitaciones para generar impactos significativos en este ámbito. Ello no compensa la falta de una política de Estado en esta materia. Por el contrario, las políticas "parciales" conciben la formación continua como un proceso desligado operacional y sistémicamente del funcionamiento del sistema educativo: sea desde la formación inicial, como de los diagnósticos sobre las fortalezas y debilidades de los docentes, la docencia, el funcionamiento del mercado docente, etc. Tampoco se vislumbran mecanismos para relacionar sinérgicamente estos componentes ${ }^{6}$, provocando una falta de perspectiva compartida sobre el sentido y rol de la formación continua en los principales actores institucionales públicos en términos de su incidencia en la profesionalización y por ende, de su impacto en los resultados educacionales.

\section{Soporte del sistema educativo en el plano institucional}

El año 2008 marcó un giro relevante en las políticas de gestión y en los instrumentos impulsados por el Ministerio de Educación hacia los establecimientos de enseñanza obligatoria. Este es un hecho no menor y responde a una crisis de magnitud de la forma cómo el Estado concibe y define su actuar en el sector educación, proceso que ha venido "postergándose y evadiéndose" precisamente por la vigencia de dos modelos de gestión del sistema educacional, que al final se tornan incompatibles, aunque este punto, aún en la actualidad no es vislumbrado por los responsables del Ministerio como de esa magnitud, y pretenden que será resuelto por la reorientación, precisamente mediante la incorporación de nuevos instrumentos de gestión de la educación obligatoria.

Una de las primeras tareas del Gobierno democrático en la década de los 90' implicó detener el proceso de reducción del tamaño del Estado y dio comienzo a

\footnotetext{
${ }^{6}$ Esta condición es difícil de superar en un enfoque de mercado "ortodoxo" como el vigente en Chile. Su solución requiere romper la fe "dogmática" en el mercado y asumir que uno de sus fallos en educación es la débil capacidad que muestra para relacionar estos componentes sin generar externalidades negativas importantes hacia la población que más depende de la educación pública.
}

Ensaio: aval. pol. públ. Educ., Rio de Janeiro, v. 17, n. 64, p. 421-448, jul./set. 2009 
una modernización en su accionar que fundamentalmente buscaba regular con éxito dispar- muchos de los cambios que ya se habían impulsado en materia económica, a saber: disciplina fiscal, control del gasto, manejo de tipo de cambio, liberalización del sistema financiero, apertura a la inversión extranjera, privatización de empresas públicas, y las reformas al Estado, siendo estas últimas posibles de analizar considerando las tres perspectivas analíticas que coexisten respecto de la temática: la administrativa, los cambios institucionales y la reforma democrática (TOMASSINI; ARMIJO, 2002).

Especificamente la reconstrucción del sistema educativo público fue asumida desde los inicios del proceso democrático como una tarea que no podía volver atrás, en términos de algunos aspectos ya consolidados: "El gobierno de la transición a la democracia tomó la decisión estratégica de no revertir el proceso de municipalización ni cambiar el modelo y mecanismos de financiamiento establecidos en 1981" (OCDE; CHILE, 2004, p. 20), con ello las autoridades del nuevo Gobierno sabian que no estaban respondiendo a las expectativas de los docentes $y$, de alguna forma validando los cambios generados en un régimen autoritario. "Tratar de reestructurar el sistema de financiamiento escolar en Chile produciría una fractura en el frágil equilibrio entre la izquierda y la derecha que formó parte implícita del acuerdo que reestableció el gobierno democrático" (OCDE; CHILE, 2004, p. 106).

Compartiendo los considerandos al respecto, son atendibles por cuando el modelo y mecanismos de operación financiera eran 'instrumentos de política educativa' tanto o más eficientes que las nuevas orientaciones políticas pro reforma educativa. En consecuencia, hacer operar una reforma educativa sobre un sistema de financiamiento asentado en otros principios, algunos incluso contradictorios, es una tarea que agregó complejidad al diseño yciertamente- también ha incidido sobre los resultados alcanzados. En lo fundamental una cosa era comprender que no se podía poner en tela de juicio algunas cuestiones estratégicas del modelo de gestión de la educación chilena, sin poner en peligro la frágil democracia recientemente conquistada, y otra distinta es creer que se podía, por la vía de la instalación de programas transversales y desligados del aparato convencional del Ministerio de Educación, resolver el conflicto entre los paradigmas en conflicto. Esto último no solamente tuvo visos inocentes sino una excesiva fe de tipo tecnocrática, como finalmente lo han demostrado los hechos actuales.

En el periodo en análisis se aplicaron diversas estrategias políticas en el accionar del Ministerio. El primer período está marcado fundamentalmente por metas reivindicativas y el reposicionamiento de la educación como un sector relevante del hacer del país. En razón de ello destacan los esfuerzos por 
incrementar los recursos públicos al sistema, mejorar las condiciones de trabajo y remuneraciones del personal docente y no docente, y comenzar un proceso de mejoría mediante proyectos específicos del equipamiento e infraestructura escolar apoyados con recursos del Banco Mundial.

Gran parte sino todas las iniciativas impulsadas son vía programas o proyectos y no se instalan como tal en la orgánica ministerial, es decir no forman parte de las tareas regulares del Ministerio, estrategia empleada para que éstas no fuesen absorbidas por la cultura organizacional vigentes. Los primeros programas son de apoyo a las escuelas con más bajos resultados escolares (conocido como P900), y luego el impulso de un programa de Mejoramiento de Calidad y Equidad (MECE), que inicialmente fue para el nivel básico urbano, extendiéndose luego al lo rural y, posteriormente a la enseñanza secundaria y superior. Las políticas en este ámbito son tipificadas como de calidad y equidad de la educación; de cambios incrementales, abiertas a la iniciativa de actores y del entorno, orientada a la escuela como eje de acción. Este tema, la escuela como centro de la acción corresponde a una visión estratégica que el Ministerio adopta además en lo programático, como una forma de romper la inadecuación del enfoque matricial 'MinisterioMunicipios', que no permite al primero (Ministerio) un adecuado control del hacer escolar más allá de algunas dimensiones importantes de carácter formal y claramente insuficientes para responsabilizarse del proceso pedagógico, como se le demanda al Ministerio, sin tener un soporte legal consistente con esta misión.

La estrategia de creación de programas para responder a demandas especificas de educación, continúa hasta el año 2000, aún cuando la Reforma Educativa es lanzada públicamente a mediados de la década de los 90, avanza esta política generando un conjunto de tensiones y de problemas que no siempre pudieron ser resueltas

Ausencia de un discurso unitario de la reforma que integre en un relato coherente y significativo el conjunto vasto de las distintas iniciativas y logre conectarlo con los temas caros a la tradición docente como: educación pública como función crucial del estado, la educación como actividad esencialmente moral -y secundariamente instrumental- rol central y de liderazgo del MINEDUC, solidaridad y no competencia, participación y no tecnocracia (GARCÍA-HUIDOBRO; COX, 1999, p. 44).

En definitiva la reforma no articula debidamente el campo de las iniciativas y se implementa un sistema de respuesta indexado en la lógica neoliberal: cada problema genera una respuesta específica o focalizada, en vez de diseñar una estrategia flexible y articuladora.

Ensaio: aval. pol. públ. Educ., Rio de Janeiro, v. 17, n. 64, p. 421-448, jul./set. 2009 
El contexto político y la necesidad de garantizar una acción gubernamental rápida llevaron a los responsables de la política educativa a desestimar, inicialmente una transformación organizacional y cultural del Ministerio de Educación, da la complejidad de esta tarea. En ese escenario, el diagnóstico sobre las capacidades del recurso humano ministerial, más los procedimientos burocrático administrativos propios de la administración pública, reticente a modificaciones, cambios de prioridades y ritmos de trabajo, llevó a las autoridades de la época a diseñar la estrategia de "programas", como una acción externa al Ministerio, pero vinculada fuertemente a su quehacer, incluso proyectando su inclusión simbólica, como parte de la "nueva institucionalidad" ministerial.

Fueron características de esta lógica la contratación de personal externo al Ministerio con carácter de expertos, con un perfil técnico y académico, con poca experiencia en la gestión real de establecimientos bajo el nuevo entramado institucional y el diseño centralizado y la intervención directa en los establecimientos, dado justamente el entramado institucional se pensaba que la alta complejidad que requerían los dispositivos para operar a escala nacional en un sistema desconcentrado, en diversos niveles, requería de especialidades técnicas provistas por profesionales que se desempeñaban en centros independientes de investigación o universidades, y por otra parte se requería la intervención directa para mostrar prontos resultados y avances en este nuevo rol con que se quería revestir la acción subsidiaria del Estado.

En relación a la primera de ellas, tuvo dos aspectos claramente marcados, los expertos educativos y los expertos en gestión y administración. Mientras los primeros eran los encargados de determinar los contenidos y naturaleza de los materiales e intervenciones, los segundos tenían como responsabilidad articular, la acción de los programas a través de unidad de programas, con la estructura ministerial oficial y rendir cuentas a los organismos financieros internacionales que financiaban las mismas. El segundo aspecto relacionado con el diseño centralizado y la intervención directa, es valorado, en tanto permitió a las autoridades y técnicos de los programas alcanzar en un periodo de tiempo adecuado la ejecución los objetivos planteados (WORLD BANK, 1999, 2001). Así, los programas ejecutaron sus actividades, articulando las exigencias administrativas de los organismos internacionales, formuladas a mediano plazo, con la lógica de planificación presupuestaria anual que exige el Estado de Chile a su función pública.

Otro elemento del diseño centralizado que es relevante destacar corresponde a la institucionalización de esta estructura en el nivel central y a una influencia en el hacer regular de las estructuras desconcentradas del Ministerio de Educación, es decir Secretarias Regionales y Direcciones Provinciales de Educación. Es en estas instancias es donde se encontraba el personal regular del Ministerio de 
Educación, y particularmente aquéllos con llegada directa a los establecimientos, los supervisores técnico pedagógicos.

Es justamente a través de estas estructuras regulares, caracterizadas como debilitadas y de baja capacidad profesional, que los expertos intervenían directamente en los establecimientos. Las implicancias de esta modalidad de operación son varias. Los empresarios, quienes contratan profesores, directores, establecen prioridades de la política local en el contexto de los planes de desarrollo comunal, entre otras funciones relevantes, no estaban considerados por el Ministerio como actores claves y fundamentales del proceso, ya sea por la voluntad política de las autoridades para reducir el rol subsidiario del Estado e intervenir en el mundo educativo con eficiencia. Pese a que las Municipalidades se democratizaron el año 1992, no fue si no hasta el año 2006 que se estableció la obligación legal de que todos los directores de establecimientos municipalizados tuvieran una duración máxima de 5 años en su cargo con posibilidades de repostular una vez cumplido ese plazo.

Mientras el primer aspecto señalado debilita la legitimidad y eficacia de cualquier intervención, el segundo refuerza el rol del director del establecimiento como una figura jerárquica de difícil acceso y de cierta inmunidad. Debilitando ambas, la responsabilidad de las autoridades formales del sistema educativo, frente a los procesos de enseñanza-aprendizaje, objeto de las transformaciones y de las políticas de mejoramiento; como también, de los resultados de aprendizaje obtenidos por los estudiantes de esos establecimientos.

Entre los años 1990 al 2007, la acción de los programas de mejoramiento no universales, puso su foco de atención en los establecimientos "focalizados". Así puede explicarse que de los más de once mil (11.000) establecimientos escolares del país, en más de 10 años de programas focalizados el universo de establecimientos alcanzado a través de estas iniciativas no alcanza una cobertura completa del sistema, al menos de aquel con subvención estatal. Un estudio encargado por el propio Ministerio de Educación en el año 2001 (CHILE, 2001), alcanza hallazgos sorprendentes como que el propio Ministerio no disponía de un catastro completo de la totalidad de iniciativas, tanto propia como de órganos del sector público que intervenían en los establecimientos. El estudio logró identificar 29 programas y elaboró una ficha de caracterización de cada uno de ellos.

Entre las debilidades identificadas por los actores se destacan aquellas relacionadas con el rol de la supervisión ministerial, señalando dos tipos de debilidades, información confusa: centrada en los procesos administrativos y plazos, compartimentada y débil articulación entre los distintos niveles y actores del Ministerio; y calidad de la relación informativa: centralista, verticalista, que culmina

Ensaio: aval. pol. públ. Educ., Rio de Janeiro, v. 17, n. 64, p. 421-448, jul./set. 2009 
poniendo el énfasis en una mirada instrumental, centrada en el quehacer del programa y las líneas de acción de estos, en detrimento de una mirada o visión centrada en la unidad básica del sistema educativo, el establecimiento escolar

El estudio de Asesorías para el desarrollo (CHILE, 2001), releva la existencia de una relación directa positiva entre los puntajes SIMCE alcanzados por los establecimientos en $8 v o$ básico y 2 do medio y las características que asume la gestión escolar: más liderazgo, más trabajo en equipo, mejor clima laboral, etc. Estudios y trabajos posteriores corroboran tales hallazgos.

En ese contexto y hacia fines de los 90, la evidencia sistemática de un impacto limitado de la lógica intervencionista centralizada, de la lógica programática, hacía reconocer explícitamente la necesidad de contar con establecimientos con autonomía, proyecto propio y capacidad de gestión, como base para la construcción de una capacidad de las escuelas de asumir su responsabilidad de manera creativa y con solvencia. Por ello, a principios del 2000, los responsables de la política pública educativa, tradujeran en un mensaje dirigido a la supervisión, la necesidad de la política pública, a través de la acción de los supervisores acompañando los mejoramientos escolares en su ruta hacia la autonomía, nominando a esta etapa: llevando la reforma al aula (COX, 2003; GARCÍA-HUIDOBRO; BELLEI, 2003). Independientemente que esto haya sido resignificado por los programas y supervisores como la necesidad de entrar fisicamente a la sala de clases, se estaba reconociendo que las estrategias implementadas durante la década de los 90 habian tocado techo en la búsqueda de un incr-emento de la calidad y la equidad en el sistema escolar chileno.

A principios del 2000, instalado este conocimiento, los programas escasamente modifican su acción, aún cuando hay cambios importantes en las autoridades ministeriales. Se consolida su institucionalización a través de su transformación en niveles y modalidades, en los que la mayor parte de las estrategias programáticas mantuvo sus líneas de acción, a veces con cambios de nombres o denominaciones.

Tomando en cuenta estos desafíos, el Ministerio de Educación impulsa el desarrollo de una estrategia de política basada en el mejoramiento continuo de la gestión de Ios establecimientos educativos: el Sistema de Aseguramiento de la Calidad de la Gestión Escolar (SACGE). La estrategia tuvo el propósito introducir practicas de mejoramiento continuo en los establecimientos a través del desarrollo de procesos de diagnóstico y autoevaluación; más una evaluación externa que entrega recomendaciones y orienta la elaboración de planes de mejoramiento. Durante el año 2003, se realizó la marcha blanca de este sistema en 68 establecimientos. Al año 2006, sobre 1300 establecimientos habian realizado procesos de diagnóstico institucional y formulado planes de mejoramiento. 
Desde el segundo año de implementación de esta política de mejoramiento, que consideraba una adhesión voluntaria de los sostenedores y que no aseguraba la entrega de recursos, demandando un tiempo de preparación que suponía una gran inversión del establecimiento, el foco estuvo puesto en los sostenedores municipales, que ya concentraban una gran cantidad de establecimientos focalizados. Esta decisión fue concientemente orientada, y se esperaba, por parte del equipo responsable del diseño la instalación de ciertas tensiones y conflictos al superponer ambas lógicas de trabajo.

El problema que se generó es que entraron en conflicto los programas focalizados con las innovaciones en gestión. Parte de esos conflictos tienen su origen en la intención de desarrollar una mirada integral de la escuela, dada por una concepción de sistema y traducida en una práctica que asegure que los procesos no se abordan aisladamente. Este principio de integralidad considera el cambio educativo como un proceso institucional que involucra a todo el centro escolar. Es decir, como un proceso dinámico donde todas las dimensiones de la escuela están relacionadas y dependen entre sí. El modelo de cambio refiere al mejoramiento continuo en organizaciones que aprenden, y por tanto a la instalación de una cultura evaluativa basada en evidencias. Cada proceso identificado es posible de ser mejorado, y cada escuela o liceo tiene "caminos" propios e irrepetibles de mejoramiento, por eso no hay recetas para el cambio, ni trayectorias preestablecidas.

Esta lógica, entró en conflicto implícito con los centros generadores de programas, dado que evidenciaba, el débil acoplamiento entre la demanda real de los establecimientos y el subsidio a la oferta del modelo ministerial de elaboración de política pública llamado de intervención.

Esta opción de política enfocó la acción de las escuelas para el mejoramiento en las variables identificadas por el Modelo de Gestión Escolar: el liderazgo ejercido principalmente por los equipos directivos y de gestión de cada establecimiento, como un elemento dinamizador y generador de cambio; la gestión curricular, entendida como el proceso principal del establecimiento vinculado con la organización, implementación y evaluación de la propuesta curricular del establecimiento, la convivencia y apoyo a los estudiantes, la organización de los recursos y la importancia de la evaluación de la calidad de los procesos implementados.

Esta forma de "mirar" los establecimientos introdujo también conflictos con la compartimentación habitual de la lógica de diseño centralizado, por nivel o subsectores de aprendizaje. Pues la modalidad de implementación y los dispositivos de diagnóstico y planificación del mejoramiento no establecian diferencias según tipos de establecimientos, ni generaba espacios artificiales para la "intervención" de los programas centrales. Este proceso de cambio en gestión se detiene 
abruptamente a mediados del año 2008. El equipo ministerial a cargo de este proceso es desmantelado y las nuevas autoridades implantan reinstalaron la visión de programas focalizados, con algunas incrustaciones menores que permanecen, pero con otra orientación.

De esta forme en materia de gestión destacan los siguientes instrumentos como aspectos claves que han quedado insertos (no necesariamente instalados) en los centros de enseñanza obligatoria:

- El marco para la buena dirección; que provee fundamentos teóricos y orientaciones de política de gestión para el centro escolar y los equipos directivos en áreas de gestión clave.

- La subvención preferencial y el desarrollo de planes de mejoramiento de los centros escolares, que vinculan instrumentos de gestión con estrategias de fortalecimiento del centro.

- El SACGE, que tuvo un impacto parcial pero que puede ser reinstalado en el sistema.

- Los planes de mejoramiento de los centros escolares, propuestas de desarrollo con un horizonte de 4 años en torno de los cuales se orientaran las acciones y recursos para el período señalado.

- La evaluación docente, proceso nacional de suma complejidad que se encuentra en su tercer año de desarrollo masivo, con implicancias aún limitadas, pero que en el largo plazo debieran ser relevantes.

\section{Estrategias de adecuación de los establecimientos escolares}

El en plano de los centros de enseñanza obligatoria, se han implementado diversas estrategias de respuesta y adecuación a las problemáticas del escenario descrito.

\section{Problemática 1:}

Sistema de financiamiento incompatible con las tareas de largo plazo que asume la educación. Ello ha ocasionado severas dificultades de financiamiento de los sistemas escolares que trabajan con la población más vulnerable, y una gran incertidumbre en las tareas de mediano y largo plazo.

Las respuestas a esta situación varían según el tipo de propiedad (dependencia) del centro educativo.

En los centros de origen privado que trabajan con recursos públicos sortean esta dificultad -principalmente- con dos estrategias bastante generalizadas y una tercera algo más selectiva: 
(i) Primero, incorporan el máximo de alumnos permitidos por cada aula (45) de forma de obtener el máximo de subvención, y de manera concomitante, muchos de los centros implementan procesos de selección de estudiantes, algo que la nueva ley de reciente aprobación (SEP) reduce para los primero seis grados de enseñanza primaria,

(ii) la selección se realiza por calificaciones escolares, lo que se asocia en grado importante a la condición socioeconómica de la población, de forma que el proceso de financiamiento ha derivado en una segregación social mayor donde los estudiantes más pobres son sistemáticamente excluidos (el pago por estudiante era prácticamente el mismo, de forma que no habian incentivos financieros mayores para trabajar con la población más vulnerable, algo que la nueva Ley (SEP) corrige parcialmente, toda vez que si bien otorga un financiamiento adicional, lo hace para un número determinado de estudiantes, contra el compromiso del establecimiento por alcanzar un determinado conjunto de metas, no siempre acordes con los recursos disponibles.

Finalmente una estrategia menos generalizada, pero de todas formas de creciente universalización en este tipo de establecimientos, es pasar a financiamiento compartido, modalidad que permite un cobro adicional a los padres, debidamente regulado en tramos, proveyendo recursos al centro escolar.

Este proceso también cuenta con un régimen laboral muy diferente si el propietario del centro Educativos es una persona jurídica privada que una pública. En el caso de los privados se rigen las contrataciones del personal por la legislación común a toda empresa de esta naturaleza, pueden fijar la remuneración sin arreglo a una escala de sueldos, y pueden contratar y despedir sin mayores argumentos, salvaguardando el régimen anual de pagode remuneraciones y de contratos que obliga la ley. Esta situación caracteriza al sector de educación subvencionada (salvo empresas de tamaño mediano y mayor), como de gran precariedad laboral, alta rotación de maestros, tratando de incorporar a los que significan gravar menos la planilla de pago.

En el caso de los centros de dependencia municipal, la situación es diferente, además porqué los maestros están sujetos al Carrera Docente, que genera un conjunto de obligaciones contractuales para el empleador público, las que derivan en un costo muy superior de la planilla de personal para estos establecimientos, mayor estabilidad funcionaria, y mayor data del personal. En lo fundamental el déficit que se produce se financia con recursos del Municipio provenientes de otras fuentes.

(i) Este tema ha sido un factor de permanente conflicto, primero con los empresarios privados que aducen que el sector público genera un mercado

Ensaio: aval. pol. públ. Educ., Rio de Janeiro, v. 17, n. 64, p. 421-448, jul./set. 2009 
poco transparente en esta materia y competencia desleal, y segundo, denuncia que los gobiernos locales imputan al presupuesto de educación personas y acciones que no están vinculadas al tema, ello por un a materia de congelamiento de las plantas de funcionarios municipales que data ya por más de 15 años.

(ii) También en forma creciente, los mismos municipios de ciudades intermedias y mayores han especializado sus establecimientos escolares para captar diversos segmentos de población, generando un sector competitivo con los mejores establecimientos particulares subvencionados, ciertamente empelando procesos similares de selección, tamaño de los cursos, etc.

\section{Problemática 2:}

Gran heterogeneidad social, reforzada por una importante segregación territorial, producto de un sistema de financiamiento que no ha sido capaz de establecer valores de subsidio asociados a los costos reales de la formación de la población más vulnerable, derivando en un incremento de la homogeneidad social al interior de cada establecimiento pero en un aumento de la heterogeneidad respecto de otros., provocando severas dificultades de socialización en la sociedad chilena (construcción de un pacto social incluyente).

Esta problemática es una de las menos desarrolladas por los centros escolares. En el caso de los particulares -salvo algunas congregaciones religiosas- es un tema omitido, se trata de problema propio del mercado, y de orientación de corto plazo, sin capacidad de anticipación estratégica:

(i) los establecimientos privados se concentran en alcanzar equilibrio financiero $y$,

(ii) en obtener buenos resultados en las pruebas nacionales de logro. Para lo cual recurren a las estrategias señaladas en la problemática anterior (1) y eventualmente en materia de buenos logros a entrenamiento en las pruebas y selección de los estudiantes.

En el caso de los centros públicos este problema tiene distinto grado de visualización.

(i) Algunas comunas grandes (50) poseen proyectos educativos más sólidos con improntas en su hacer y con un sistema organizado de establecimientos según tipos de educación y segmento de la población.

(ii) Sin embargo en un gran número de las restantes 300 comunas este problema no existe, pues la tarea del día a día es la supervivencia financiera, lo que ha conducido al sector escolar, a atender toda la población que demande educación sin establecer filtros o procesos de selección, siendo efectivamente este sector el que cumple el derecho constitucional de la provisión de educación para toda la población. 
Problemática 3:

Dificultades relevantes para implementar alianzas y estrategias de largo plazo entre diversos actores intra- inter sistema educativo, producto del sistema de concesiones, licitaciones y otros implementados bajo mecanismos de mercado. Siendo concordante con la visión de Estado subsidiario, el Ministerio de Educación no puede generar alianzas trascendentales con socios estables en los distintos procesos que debe afrontar, en especial en los de formación continua.

El Ministerio no ha podido generar un sistema de estímulos relevantes que impliquen asociatividad de empresarios públicos y privados. Por el contrario, sus principales mecanismos impulsan la competencia, incluso entre las unidades educativas de un mismo empresario. Esta situación da cuenta de un irracional "invisible" para la autoridad nacional y subnacional. Si bien no hay mecanismos que "impidan" la cooperación, la situación desigual que se produce, a raíz de las restricciones legales de las entidades públicas ante las privadas, hace que en los hechos ella no ocurra y los escasos intentos que se han impulsado, van camino al fracaso. La resultante de esto es falta de alianzas de mediano y largo plazo, cambios constantes de proveedores, ausencia de una visión sistémica, solo la unidad educativa es el centro, aunque los empresarios finalmente no obtengan ni mayor eficiencia técnica ni financiera.

\section{Problemática 4:}

Escasa autonomía de los centros escolares municipales y de sus cuadros directivos para asumir decisiones relevantes en materia de selección, contrato y cambio de docentes, de manejo de recursos financieros, etc.

Esta problemática afecta solamente al sector municipal, atendiendo al hecho que los privados se rigen por toda una normativa diferente que hace la situación caso a caso. En consecuencia, la problemática señalada en parte de la normativa legal del sector. Si bien existe un procedimiento que se denomina "delegación de funciones" en la cual la autoridad comunal de educación puede traspasar algunas de sus responsabilidades al Director del establecimiento escolar, en lo fundamental estas se refieren a la gestión operacional y de gastos corrientes del establecimiento, sin considerar la gestión financiera, ni las remuneraciones del personal, ni las partidas presupuestarias mayores.

Esta situación es un tema de cultura organizacional, de estructuración del sistema público, de prácticas (hábitos) enraizadas en una tradición de que la autoridad no tiene tuición sobre contratos y destinaciones de personal. Sólo le compete la gestión del currículo. Ello ha impedido que los establecimientos se responsabilicen por los resultados, más allá de las normativas como el SACGE y otras, por cuanto los docentes no dependen directamente al Director en materia contractual para permanecer en su cargo, existiendo e autoridad sin responsabilidad, afectando con ello el desarrollo del sistema público en su conjunto. 
Lo interesante es que el conjunto de instrumentos desarrollados para la buena gestión de las comunas, como es el Plan de Desarrollo Educativo Comunal (PADEM), y los Planes de Desarrollo Educativo por establecimiento, son documentos que teniendo validez legal, pueden ser modificados en reiteradas veces de manera que su constante adecuación termina siendo un factor atentatorio con la estabilidad y orientación de la acción y suelen ser más bien instrumentos que "se ajustan" a lo que finalmente termina ocurriendo en las unidades educativas públicas, entre otros motivos, por el hecho que le Ministerio carece del soporte legal para poder adoptar otras medidas.

\section{Problemática 5:}

Aumento de visiones tecnocráticas de la gestión, desligadas de la práctica educativa de los centros escolares. Se enfatiza la eficiencia en el manejo de los recursos como clave del éxito como un factor independiente del capital social de los estudiantes y sus familias, y de las condiciones del trabajo laboral docente, atribuyéndose a los privados la mayor eficiencia, más que a las condiciones bajo las cuales éstos trabajan.

En la actualidad el énfasis en los procesos de gestión de los establecimientos escolares, referidos en lo fundamental a la variable resultados de logros escolares en las pruebas respectivas de nivel nacional (SIMCE de 4,8 y 10 grado) e internacionales (PISA), es el baremo tras el cual se comparan todos los resultados educacionales. A parte de ser un enfoque reduccionista, usualmente este proceso posee los siguientes vicios:

(i) se igualan las comparaciones por dependencia del empresario (Part. Pagado, Part. Subvencionado y municipal) y no por el capital social y cultural de la población,

(ii) al omitirse las diferencias de capital social, los grupos aparecen como homogéneos, no siéndolo, teniendo regularmente mejores resultados los establecimientos de naturaleza particular, entre otros motivos por las estrategias de sobrevivencia señaladas en la problemática 1,

(iii) se extrapolan los resultados al extremo que resulta evidente privatizar, pero al controlarse por capital social y cultural se parecen mucho los resultados, en algunos casos no hay diferencias significativas y en otros son favorables a los públicos,

(iv) poniéndose de esta forma en tela de juicio que el valor agregado de los establecimientos, sobre el capital social y cultural es reducido, materia que no se explicita debidamente "fortaleciendo la visión tecnocrática del agestión" como un acto desligado de las condiciones basales de trabajo y de las características de la población.

\section{Problemática 6:}

Ausencia de una carrera docentes vinculante con la formación inicial y permanente. Falta de incentivos de desempeño y de recompensa. 
Uno de los problemas más complejos que posee el sistema educacional chileno es la falta de regulaciones de carácter vinculante entre la formación inicial, la formación de carácter continua, los requerimientos derivados del currículo escolar hacia la profesión docente, y el mercado laboral, a saber: remuneraciones y carrera docente, lo que además se traduce en normativas, requisitos y criterios diferentes según sea el empresario: público o privado.

El origen de este problema se sitúa en las reformas del año 1981. A la fecha no han sido corregidas bajo ninguna medida efectiva y son consustanciales a la aplicación de la economía de mercado, y ciertamente, a todo el sistema educacional chileno. En razón de ello, las reformas al mercado de trabajo del año 1976 se tradujeron en que los docentes que laboran en el sector privado lo hacen bajo las leyes de cualquier empresa de esta naturaleza, con la salvedad (establecida por ley hace pocos años) que los contratos deben ser anuales por el período del año escolar (marzo a febrero del año siguiente). Antes de esta normativa, en el sector particular algunos docentes tenía contratos mensuales.

Con la reforma al sistema educativo del año 1980 y el traspaso de los establecimientos y de los docentes al sistema municipal, se precarizó el empleo docente. Primero se produjeron despidos masivos en esos años para ajustar las "plantas docentes a los requerimientos presupuestarios" " y segundo, el empleador es ahora el alcalde y no el Estado directo, además que existir una normativa confusa en algunas materias, que para aciertos efectos no son empleados públicos y para otros si lo son, materias que se han ido resolviendo mediante jurisprudencia. De todas formas esta reforma desligó absoluta y completamente el ejercicio de la profesión con los aspectos de formación inicial, requerimiento del currículo y formación continua, y más con los aspectos de carrera docente, estableciendo uno de los principales nudos críticos que el país no ha sabido resolver (DONOSO, 2008).

En forma complementaria, la formación inicial redujo sus exigencias desde el año 1980 hasta 1995, de manera que la formación de profesores perdió su rango universitario por una década y media, recuperándolo en 1995 pero sin cambiar lo ocurrido. No existe un nexo formal entre las instituciones formadoras de docentes, con las nuevas exigencias del currículo, los nuevos proyectos de formación inicial docente y las exigencias del mercado laboral (ÁVALOS, 2003) Las nuevas exigencias. Esta falta de sincronía genera uno de los principales problemas que en gestión del currículo se pueden enfrentar, a saber:

\footnotetext{
${ }^{7}$ Durante la década de los 80 (1981 -9889) el presupuesto del sector público asignado a educación se redujo en un 24\% en términos reales respecto de 1981 (COX, 2003).
}

Ensaio: aval. pol. públ. Educ., Rio de Janeiro, v. 17, n. 64, p. 421-448, jul./set. 2009 
(i) El mercado laboral privado y público se rige por criterios y procedimientos distintos, casi no hay un mercado sino submercados (ALMONACID; LUZÓN; TORRES, 2008), con consecuencias importantes al respecto.

(ii) La formación inicial de docentes no está ligada a las exigencias del currículo prescrito vigente, ni tampoco a mecanismos eficientes de inserción laboral.

(iii) No hay nexo significativo algunos entre el mercado docente privado y público, no hay vinculación que establezcan un piso de calidad.

(iv) Desde el año 2008 se aplica un examen de resultados a los estudiantes d pedagogía, aún sin claridad respecto de sus consecuencias, ni menos acerca de sus impactos sobre la calidad de los aprendizajes de los futuros profesores.

(v) La formación continua no posee ningún nexo explícito con la carreraza docente.

(vi) El mercado laboral docente no da cuenta de diferencias relevantes de calidad de los docentes, sea por su excesiva territorialización como por su segmentación espacial.

(vii) Mientras no exista un sistema integrado de carrera docente, con criterios equivalentes y vinculantes con la formación inicial, continua un sistema de incentivos es complejo poder intentar mejoras con impacto en estas materias.

\section{Problemática 7:}

El sistema de renovación de equipos directivos de los centros públicos posee al menos dos problemas relevantes para ser eficiente: (i) requiere de financiamiento para operar adecuadamente, para indemnizar a los directivos que cesan en sus funciones; (ii) no existen incentivos suficientes para que postulen a los nuevos cargos personas competentes.

Teniéndose claro que uno de los principales problemas del sector público fueron las leyes de amarre en educación dejadas por la Dictadura cívico/militar (Ley Orgánica de Enseñanza e inamovilidad de los directivos). Si a comienzos de la presente década se logró destrabar este proceso con una ley que permite la reconcursabilidad cada 5 años de los directivos, bajo ciertos procedimientos muy amplios, lo cierto que el principal escollo de la ley ha sido:

(i) su falta de financiamiento, de manera que no se ha podido cambiara las personas porque los municipios no disponen de los recursos para indemnizarles, y segundo que el sistema de concursos ha sido muy poco convergente con los requerimientos de los cargos.

(ii) Adicionalmente, dado que los cargos son por un período de 5 años, contra un proyecto educativo, muchos docentes no se interesan pues ello implica perder la propiedad del cargo actual. 
(iii) Correlativamente se sabe poco de la evaluación que se ha hecho de las propuestas y resultados alcanzados en los casos en que los directivos ha reconcursado y finalizado sus períodos, montando con ello un problema severo de información que posee el sector.

En un anuncio reciente a nivel presidencial (21/05/2009) se plantea un programa de formación de 2000 directivos escolares, sin embargo no queda claro en el texto no solamente como serán formados sino de su origen, si están insertos ya ene I sistema o si serán insertados, cuáles serán los mecanismos que se emplearan al respecto.

\section{Problemática 8:}

Los resultados de la evaluación docente y el mercado laboral docente del sector público.

Desde el año 2007 se ha venido evaluando a distintos grupos de docentes que trabajan en el sector público. Si bien la evaluación posee impacto en los casos negativos, el problema es que no es vinculante con los aspectos positivos (más allá de mantener el cargo) ni conlleva tampoco con orientaciones derivadas de este procesamiento para el efectivo mejoramiento de la docencia, excepto en aquellos casos que han sido evaluados negativamente.

\section{Palabras finales}

La gestión de los centros de enseñanza obligatoria en el caso de Chile presenta un conjunto de nudos críticos derivados del conflicto no resuelto a la fecha entre la implantación de políticas de mercado con otras propias del Estado de Bienestar. Este debate traspasa el conjunto del sistema educacional y se expresa en el área de gestión en aspectos claves, como un sistema de financiamiento complejo, que ha precarizado el funcionamiento del sistema escolar y que ha obstaculizado la posibilidad de generar alianzas estratégicas entre el Ministerio de Educación y los establecimientos con horizonte de mediano y largo, con recursos sustentables en el tiempo.

En segunda instancia y derivado de esta situación, hay marcadas diferencias en materia de gestión entre los centros de enseñanza obligatoria de origen particular con aquellos de carácter municipal. Esto se expresa en aspectos claves como la forma como funciona la carrera docente y su nexo con el mercado laboral, y en un segundo nivel, por los escasos vínculos que se generan entre la formación inicial y permanente de los docentes, los requerimientos laborales y la gestación de mercados y submercados en educación.

El Ministerio de Educación se encuentra en un proceso de actualización que ha tenido aciertos y desaciertos en el campo de la gestión, la que avanzó con nuevos

Ensaio: aval. pol. públ. Educ., Rio de Janeiro, v. 17, n. 64, p. 421-448, jul./set. 2009 
aires entre los años 2000 al 2007 instalando un conjunto de mecanismos e instrumentos orientados a generar una nueva cultura en este ámbito, sin embargo este proceso se detuvo abruptamente el año 2008, siendo sustituido por otras visiones que se orientan a fortalecer la gestión en el aula. Algunos aspectos claves en este sentido no han sido resueltos, uno es la escasa autonomía que poseen los directivos de los centros municipales en la mayor parte de las dimensiones de gestión, insuficiente financiamiento público de la educación, un soporte legal muy rudimentario y obsoleto para tareas que demanda un funcionamiento más ágil y respuesta oportunas y de fondo a demandas claves.

\section{Referencias}

ÁVALOS B. La formación de profesores y su desarrollo profesional. Prácticas innovadoras en busca de políticas. In: COX, C. (Ed.). Políticas educacionales en el cambio de siglo: la reforma del sistema escolar en Chile. Santiago, Chile: Editorial Universitaria, 2003. p. 559-594.

ALMONACID, C.; LUZÓN, A.; TORRES, M. Cuasi mercado educacional en Chile: el discurso de los tomadores de decisión. Archivos Analíticos de Políticas Educativas, St. Petersburg, FL, v. 16, n. 8, 2008. Disponible en: <http:// epaa.asu.edu/epaa/v16n8/>. Consultado en: mayo 2008.

BECA, C. La política de formación continua de docentes: avances y desafíos. In: ARELLANO, M.; CERDA, C. (Ed.). Formación continua de docentes, un camino para compartir 2000 -2006. Santiago de Chile, CPEIP, 2006. p. 19-31.

BRUNNER, J. J.; ELACQUA, G. Capital humano en Chile. Santiago, Chile: Universidad Adolfo lbáñez, 2003.

CHILE. Consejo Asesor Presidencial para la calidad de la educación. Informe final. Santiago, Chile, dic. 2006. Disponible en: <www.presidencia.cl>. Consultado en: dic. 2006.

CHILE. Ministerio de Educación. Asesorías para el Desarrollo. Análisis de la oferta de programas e iniciativas del sector público y privado desde los establecimientos educacionales. Santiago, Chile, 2001.

CHILE. Ministerio de Educación. Asesorias para el Desarrollo. Efectividad escolar y cambio educativo: en condiciones de pobreza en Chile. Santiago, Chile, 2007.

CHILE. Ministerio de Educación. Indicadores de la educación en Chile 2002. Santiago, Chile, 2002. 
CHILE. Ministerio de Planificación. Ministerio de Educación. Educación y pobreza: resultados de la encuesta Casen 2003. Santiago, Chile, 2004. Disponible en: <www.mideplan.cl/casen>. Consultada en: 27 oct. 2004.

COX, C. Las políticas educacionales de Chile en las últimas dos décadas del siglo XX. In: COX, C. (Ed.). Políticas educacionales en el cambio de siglo: la reforma del sistema escolar en Chile. Santiago, Chile: Editorial Universitaria, 2003.

DONOSO, S. El perfeccionamiento docente en Chile (1990 -2007): ¿estado versus mercado?. Revista Brasileira de Educação, Rio de Janeiro, v. 13, n. 39, p.437454, 2008.

Reforma y política educacional en Chile 1990-2004: el neoliberalismo en crisis. Estudios Pedagógicos, Valdivia, Chile, v. 31, n. 1, p.113-135, 2005.

GARCÍA-HUIDOBRO, J. E.; BELLEI, C. Desigualdad educativa en Chile. Santiago, Chile: Universidad Alberto Hurtado, 2003.

GARCÍA-HUIDOBRO, J. E.; COX, C. La reforma educacional chilena 1990-1998: visión de conjunto. In: GARCÍA- HUIDOBRO, J. E. (Ed.). La reforma educacional chilena. Madrid, Editorial Popular, 1999.

HERRERA, M. 0.; BELLEI, C. ¿Qué se sabe sobre la calidad de la educación parvularia chilena? Santiago, Chile: Universidad de Concepción: UNICEF, 2002.

MIZALA, A.; ROMAGUERA, P. Regulación, incentivos y remuneraciones de los profesores en Chile. In: COX, C. (Ed.). Políticas educacionales en el cambio de siglo. Santiago, Chile: Editorial Universitaria, 2003.

OCDE; CHILE. Ministerio de Educación. Revisión de las políticas nacionales de educación: organización para la cooperación y el desarrollo. París: Santiago, Chile, 2004.

PAVEZ, J. Situación delos docentes sus condiciones laborales y profesionales. In: HEVIA, R. (Ed.). La educación en Chile hoy. Santiago, Chile: Ediciones Universidad Diego Portales, 2003.

RAMA, G.; NAVARRO, J. C. Carrera de los maestros en América Latina. In: MAESTROS de América Latina: nuevas perspectivas sobre su formación y desempeño. Santiago, Chile: BID: PREAL, 2004.

ROJAS, P. Renumeraciones de los profesores en Chile. Estudios Públicos, Santiago, Chile, n. 71, p. $122-175,1998$. 
TOMASSINI, L.; ARMIJO, M. Reforma y modernización del Estado. Santiago, Chile: Lom Editores, 2002.

WORLD BANK. Implementation completion report. Republic of Chile: Primary Education Improvement Project (Loan 3410-CH). Washington, DC, 1999.

WORLD BANK. Implementation completion report. Republic of Chile: Secondary Education Improvement Project (Loan 3410-CH Washington, DC, 2001.

Recebido em: 17/06/2009

Aceito para publicação: 10/07/2009 